\section{Neurogenic bladder dysfunction in children}

In children, neurogenic bladder dysfunction is managed medically and using various surgical approaches. A recent study by Guys et al. has investigated whether the technique of sacral neuromodulation (SNM) may be useful in this setting.

A total of 42 patients aged 5-21 years were included in the study. After testing for appropriate responses to stimulation of the S3 nerve, patients were randomized to SNM or conventional treatment, with 21 patients in each group. Patients randomized to SNM underwent implantation of the permanent neurostimulator unit and lead immediately after testing. Those in the conventional treatment (control) group received anticholinergics, antibiotics and bulking agents as necessary, but were not treated surgically during the study period. Patients were evaluated at 3, 6, 9 and 12 months.

There were no statistically significant differences in urodynamic variables between the SNM and control groups, with two exceptions: better bladder capacity in the control group at 12 months, and higher leak point pressure in the SNM group at all time points. Patients in the SNM group showed significant improvements in compliance and functional bladder capacity at 6 and 9 months, compared to baseline. Other than three cases of revision surgery (resulting from lead migration, faulty connection or wound infection), there were no complications associated with the SNM procedure.

Although conclusions could not be drawn regarding the potential benefit of SNM, the authors note that the results were encouraging and that a larger study is ongoing.

Original article Guys JM et al. (2004) Sacral neuromodulation for neurogenic bladder dysfunction in children. J Urol 172: 1673-1676

\section{Abnormal urinary cytology following flexible cystoscopy}

Abnormalities in voided urine cytology, including papillary aggregration and enlarged nuclei, are routinely used as markers for bladder neoplasia. Similar changes can be caused by examination of the urinary tract by flexible cystoscopy. Since this method is routinely used in the diagnosis of transitional cell carcinoma of the urinary tract, it is possible that these reactive changes could lead to misdiagnosis of malignancy if urine is analyzed too soon after instrumentation. McVey et al. have carried out a study to determine how quickly after flexible cystoscopy the urine cytology returns to normal.

A total of 48 patients provided urine samples before undergoing flexible cystoscopy. Further samples were provided immediately after the procedure and at 1,2, 4, 7, 14 and 28 days. Anonymized cytology slides prepared from each sample were then examined by three cytopathologists.

As expected, samples provided immediately after cystoscopy showed a substantial increase in urothelial cells. Accompanying morphological changes included enlarged nuclei and nuclear atypia, formation of papillary clusters, and elongation of cells to form polarized, 'columnar' cells. In most cases, these cellular abnormalities returned to normal 1 day following instrumentation.

The study confirms that the process of flexible cystoscopy produces transient urinary cytological changes suggestive of malignancy. A time interval of 1 day between cystoscopy and cytological examination is usually sufficient to avoid false-positive results. Consequently, McVey et al. recommend that details about the interval from any endoscopic procedure are essential when sending urine for cytological analysis after instrumentation.

Original article McVey RJ et al. (2004) How long is urinary cytology abnormal after flexible cystoscopy? BJU Int 94: 548-551

\section{Risk factors for bladder cancer in nonsmokers}

The Los Angeles Bladder Cancer Study linked 4-aminobiphenyl exposure to the development of bladder cancer in nonsmokers. In an extension of the study, Gan and colleagues have investigated whether other arylamines are similarly involved.

This population-based, case-control study involved 298 bladder cancer patients and 308 control subjects. All subjects were interviewed about their demographic characteristics, diet, tobacco and alcohol use, employment history, medical conditions and use of medication. 